\title{
Spontaneous Scalarization of Charged Black Holes
}

\author{
Carlos A. R. Herdeiro, ${ }^{1}$ Eugen Radu, ${ }^{1}$ Nicolas Sanchis-Gual, ${ }^{2}$ and José A. Font ${ }^{2,3}$ \\ ${ }^{1}$ Departamento de Física da Universidade de Aveiro and CIDMA, Campus de Santiago, 3810-183 Aveiro, Portugal \\ ${ }^{2}$ Departamento de Astronomía y Astrofísica, Universitat de València, Carrer del Dr. Moliner 50, 46100, Burjassot (València), Spain \\ ${ }^{3}$ Observatori Astronòmic, Universitat de València, c/Catedrático José Beltrán 2, 46980, Paterna (València), Spain
}

(Received 19 June 2018; published 5 September 2018)

\begin{abstract}
Extended scalar-tensor Gauss-Bonnet (ESTGB) gravity has been recently argued to exhibit spontaneous scalarization of vacuum black holes (BHs). A similar phenomenon can be expected in a larger class of models, which includes, e.g., Einstein-Maxwell scalar (EMS) models, where spontaneous scalarization of electrovacuum BHs should occur. EMS models have no higher curvature corrections, a technical simplification over ESTGB models that allows us to investigate, fully nonlinearly, BH scalarization in two novel directions. First, numerical simulations in spherical symmetry show, dynamically, that ReissnerNordström (RN) BHs evolve into a perturbatively stable scalarized BH. Second, we compute the nonspherical sector of static scalarized $\mathrm{BH}$ solutions bifurcating from the RN BH trunk. Scalarized BHs form an infinite (countable) number of branches and possess a large freedom in their multipole structure. Unlike the case of electrovacuum, the EMS model admits static, asymptotically flat, regular on and outside the horizon BHs without spherical symmetry and even without any spatial isometries, which are thermodynamically preferred over the electrovacuum state. We speculate on a possible dynamical role of these nonspherical scalarized BHs.
\end{abstract}

DOI: 10.1103/PhysRevLett.121.101102

Introduction.-The newborn field of gravitational wave astronomy [1-6] will test the nature of astrophysical black holes (BHs) in an unprecedented way. It is therefore of the utmost importance to theoretically scrutinize all physically reasonable alternatives to the Kerr $\mathrm{BH}$ [7] hypothesis, a paradigm supported by the uniqueness theorems for vacuum general relativity (GR) [8-10].

A reasonable alternative $\mathrm{BH}$ model requires, conservatively, being a solution of a consistent physical theory and having robust dynamical properties. The latter include a formation mechanism and sufficient stability. A novel class of such models has recently emerged in extended scalar-tensor Gauss-Bonnet (ESTGB) gravity [11-13]. In ESTGB, the Schwarzschild BH may become spontaneously scalarized, since linear theory reveals it becomes unstable against scalar perturbations for sufficiently small BHs. Moreover, BHs with scalar hair exist in the model which are thermodynamically preferred over the vacuum solution [11] and stable against spherical perturbations [14]. This phenomenon is akin to the spontaneous scalarization found long ago for neutron stars in the context of scalar-tensor theories [15], but with the scalarization induced by strong spacetime curvature, rather than matter (see Ref. [16] for a discussion of matter-induced $\mathrm{BH}$ scalarization).

The studies of BH scalarization in Refs. [11-14] were both nondynamical and, for the nonlinear scalarized solutions, restricted to the spherically symmetric sector. However, the instability of the vacuum solutions observed in linear theory contains nonspherical modes. This raises the issues of what other scalarized, static BH solutions are admitted in ESTGB and what is the dynamical evolution and end point of the instability of vacuum BHs.

A complete understanding of these evolutions requires nonlinear numerical simulations; these are challenging, particularly if no symmetries are imposed, in view of the higher-curvature terms in ESTGB gravity. In this Letter we tackle these questions by observing that the ESTGB model belongs to a larger universality class, that in particular includes the technically simpler Einstein-Maxwell scalar (EMS) models [17]. In an illustrative EMS model, we perform, in spherical symmetry, nonlinear numerical simulations exhibiting, dynamically, the scalarization of the Reissner-Nordström ( $\mathrm{RN}) \mathrm{BH}$. The process indeed forms a perturbatively stable scalarized $\mathrm{BH}$. In the same EMS model, we study the nonspherical, static, scalarized solutions, and show they are thermodynamically preferred over the (electrovacuum) spherical BH. Since in cousin models to EMS, nonlinear numerical evolutions of binary BHs have been performed [22] (see also Refs. [23-26]), even nonspherical, nonlinear evolutions of the scalarization instability in EMS models are within reach.

Besides exhibiting, dynamically, the scalarization instabilty in the spherical sector, our investigation of the static nonspherical sector constructs the first examples of static, asymptotically flat, regular on and outside the event horizon BHs without spatial isometries. This is a maximal violation of Israel's uniqueness theorem [27] stating that 
static BHs in electrovacuum must be spherical. The nonminimal couplings in EMS models circumvent in a radical way the uniqueness and simplicity of (electro)vacuum BHs [10], which holds even if a minimal coupling to a real scalar field is included [28,29]. It endows BHs with multipole freedom.

Universal conditions and a toy model.-Consider the following scalar field action on a nontrivial background (e.g., a curved spacetime and/or electromagnetic field):

$$
\mathcal{S}_{\phi}=-\int d^{4} x \sqrt{-g}\left[2 \partial_{\mu} \phi \partial^{\mu} \phi+f(\phi) \mathcal{I}(\psi ; g)\right],
$$

where $f(\phi)$ is the coupling function and $\mathcal{I}$ is a source term which generically depends on the matter field(s) $\psi$ and the metric tensor $g_{\mu \nu}$. The scalar-field equation of motion is $\square \phi=f^{\prime} \mathcal{I} / 4 . f(\phi)$ must allow the existence of a scalarfree solution, with $\phi=0$. This requires $f^{\prime}(0)=0$. Spontaneous scalarization occurs if the scalar-free solution is unstable against scalar perturbations $\delta \phi$. These obey $\left(\square-\mu_{\text {eff }}^{2}\right) \delta \phi=0$, where $\mu_{\text {eff }}^{2} \equiv f^{\prime \prime}(0) \mathcal{I}$ (background) $/ 4$. If $\mu_{\text {eff }}^{2}<0$, a tachyonic instability sets in, driving the system away from the scalar-free solution.

Consider, as a field theory illustration of the instability,

$$
\mathcal{I}=F_{\mu \nu} F^{\mu \nu},
$$

where $F=d A$ is the Maxwell tensor, in Minkowski spacetime. The scalar-free configuration is the Coulomb solution (in standard spherical coordinates):

$$
\phi=0, \quad A=\frac{Q}{r} d t .
$$

Consider also (in appropriate units) $f(\phi)=\left(1-\phi^{2}\right)^{-1}$. These choices obey $f^{\prime}(0)=0$ and $\mu_{\text {eff }}^{2}<0$. Thus, Eq. (3) is unstable against scalarization. This model admits a simple closed-form spherically symmetric scalarized solution:

$\phi=\zeta \sin \left(\frac{Q}{r}\right), \quad A=\left[\left(1-\frac{\zeta^{2}}{2}\right) \frac{Q}{r}+\frac{\zeta^{2}}{4} \sin \left(\frac{2 Q}{r}\right)\right] d t$,

where the integration constant obeys $|\zeta|<1$, to avoid divergences in the coupling. The total energy of the configurations is $E=4 \pi \int_{r_{0}}^{\infty} \rho d r$, where $\rho=-T_{t}^{t}$ is the energy density. We consider a conducting sphere at $r=r_{0}$ and solutions (3) and (4) only for $r \geq r_{0}$ to regularize the total energy. The energies of these exterior configurations are, respectively, $E^{(\phi=0)}$ and $E^{(\phi \neq 0)}$. Then, $E^{(\phi \neq 0)}-E^{(\phi=0)}=$ $\pi \zeta^{2} Q \sin \left[\left(2 Q / r_{0}\right)\right]$. The scalarized solution is energetically favored in a set of bands, defined as $\left.Q / r_{0} \in \pi\right] n+$ $1 / 2, n+1\left[\right.$. The integer $n \in \mathbb{N}_{0}$, labeling the bands also counts, via Eq. (4), the number of nodes exterior to $r_{0}$ of the scalar field profile. Thus, within these bands, the instability (likely) evolves dynamically from (3) into (4).

Spontaneous scalarization of BHs. - The toy model shows that spontaneous scalarization (i) is not exclusive of gravitational models and (ii) can be supported by an electromagnetic nonminimal coupling. We shall now focus on the case of BHs and consider the gravitational model $(G=1=c)$ :

$$
\mathcal{S}=\frac{1}{16 \pi} \int d^{4} x \sqrt{-g} R+\mathcal{S}_{\phi}
$$

where $R$ is the Ricci scalar. On a spherical, scalar-free $\mathrm{BH}$ solution, with a generic line element

$d s^{2}=-N(r) e^{-2 \delta(r)} d t^{2}+\frac{d r^{2}}{N(r)}+r^{2}\left(d \theta^{2}+\sin ^{2} \theta d \varphi^{2}\right)$,

performing a (real) spherical harmonics decomposition of the scalar field $\phi(r, \theta, \varphi)=\sum_{\ell m} Y_{\ell m}(\theta, \varphi) U_{\ell}(r)$, the scalar field equation becomes

$$
\frac{e^{\delta}}{r^{2}} \frac{d}{d r}\left(\frac{r^{2} N}{e^{\delta}} \frac{d U_{\ell}}{d r}\right)-\left(\frac{\ell(\ell+1)}{r^{2}}+\mu_{\mathrm{eff}}^{2}\right) U_{\ell}=0 .
$$

Equation (7) is an eigenvalue problem: for a given $\ell$, requiring an asymptotically vanishing, smooth scalar field selects a discrete set of BHs. These are the bifurcation points of the scalar-free solution. The (test) scalar field profiles they support-hereafter referred to as scalar clouds-are distinguished by the node number of $U_{\ell}(r)$, $n \in \mathbb{N}_{0}$, besides $(\ell, m)$.

In Refs. [11-13], the model (5) was studied with $\mathcal{I}=\mathcal{L}_{G B}$, where $\mathcal{L}_{G B}$ is the Gauss-Bonnet invariant, and with various different coupling functions, satisfying $f^{\prime}(0)=0$ and $\mu_{\text {eff }}^{2}<0$ (see also Refs. [16,30]). In this case, the spontaneous scalarization of the Schwarzschild solution is induced by this higher-curvature correction, which is nontrivial for those BHs. Here, we shall take the source in Eq. (2). Then, scalarization of the ReissnerNordström (RN) BH is induced without the need of highercurvature corrections.

Scalarization in EMS models.-We are interested in models as given in Eq. (5), with Eqs. (1) and (2) which (i) admit the scalar-free $\mathrm{RN}$ solution, ruling out the usual Einstein-Maxwell dilaton model [31], where $f(\phi)=e^{-\alpha \phi}$, and (ii) approach the standard Einstein-Maxwell system in the far field, i.e., $\phi \rightarrow 0$ and $f(\phi) \rightarrow 1$ as $r \rightarrow \infty$.

From the scalar equation of motion, it is possible to derive two Bekenstein-type identities [32] that set the following constraints on $f$ (for a purely electric field $\left.F^{2}<0\right): f_{, \phi \phi}>0$ and $\phi f_{, \phi}>0$ for some range of the radial coordinate $r$. A simple potential compatible with the above requirements, that we shall use hereafter, is 


$$
f(\phi)=e^{-\alpha \phi^{2}} .
$$

The coupling $\alpha$ is a dimensionless constant, and the conditions on $f$ imply $\alpha<0$ for $F^{2}<0$.

The RN (scalar-free) solution is given by Eqs. (3) and (6), with $\delta=0, N(r)=1-2 M / r+Q^{2} / r^{2}$. The scalar perturbations on this background are given by Eq. (7) with $\mu_{\text {eff }}^{2}=\alpha Q^{2} / r^{4}<0$, hence exhibiting the instability. For $\ell=0$, one finds an exact test field solution

$$
U_{0}(r)=P_{u}\left[1+\frac{2 Q^{2}\left(r-r_{H}\right)}{r\left(r_{H}^{2}-Q^{2}\right)}\right]
$$

where $u \equiv(\sqrt{4 \alpha+1}-1) / 2, r_{H} \equiv M+\sqrt{M^{2}-Q^{2}}$, and $P_{u}$ is a Legendre function. For generic parameters $\left(\alpha, Q, r_{H}\right)$, finding the $\ell=0$ bifurcation points from $\mathrm{RN}$ reduces to studying the zeros of this function as $r \rightarrow \infty$. Examples are shown in Fig. 4 below. Bifurcation requires $\alpha$ below a maximal value, $\alpha_{\max }$. For fundamental modes $(n=0)$, we obtain $\alpha_{\max }=-1 / 4(\ell=0), \alpha_{\max } \simeq-2.784$ $(\ell=1)$, and $\alpha_{\max } \simeq-7.087(\ell=2)$.

Spherical sector (no nodes): Domain of existence.-The spherical scalarized BHs bifurcate from the RN solution for any $\alpha<-1 / 4$. They are the nonlinear realizations of the $(n, \ell, m)=(0,0,0)$ clouds. These nonlinear solutions were obtained by using the ansatz (6), $\phi=\phi(r)$, $A=V(r) d t$, and standard numerical techniques to solve coupled nonlinear ODEs-see the Supplemental Material [33] (Sec. I) for details, which includes Refs. [34-37]. By varying $\alpha$, we have obtained the domain of existence of spherical scalarized BHs (with $n=0$ ) shown in Fig. 1.

For each $\alpha$, a branch of scalarized solutions bifurcates from a $\mathrm{RN} \mathrm{BH}$ with a particular charge-to-mass ratio $q \equiv Q / M$. As $\alpha$ varies, these RN BHs define an existence line. Each constant $\alpha$ branch ends at a critical, (likely) singular configuration: the numerics indicate the Kretschmann scalar and the horizon temperature diverge,

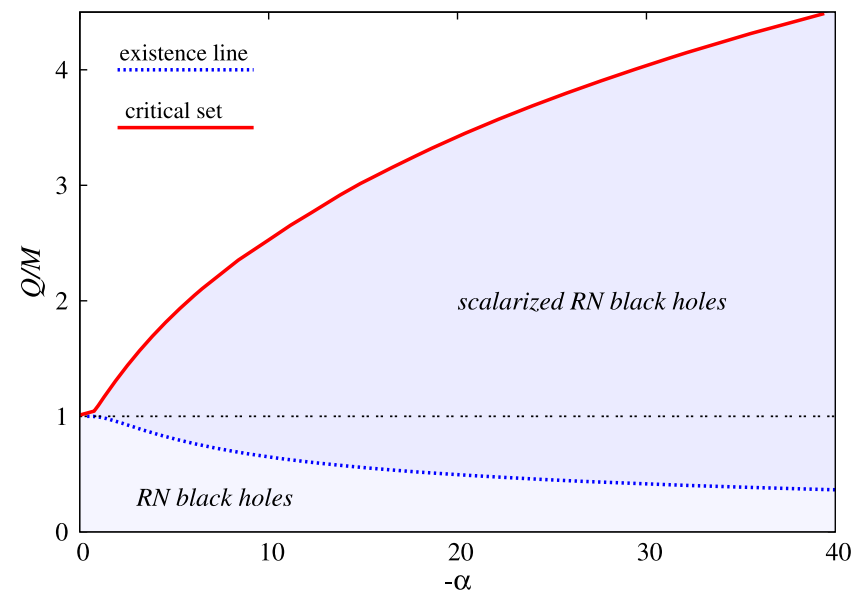

FIG. 1. Part of the domain of existence of spherical EMS scalarized BHs with $n=0$ in the $(\alpha, q)$ plane.

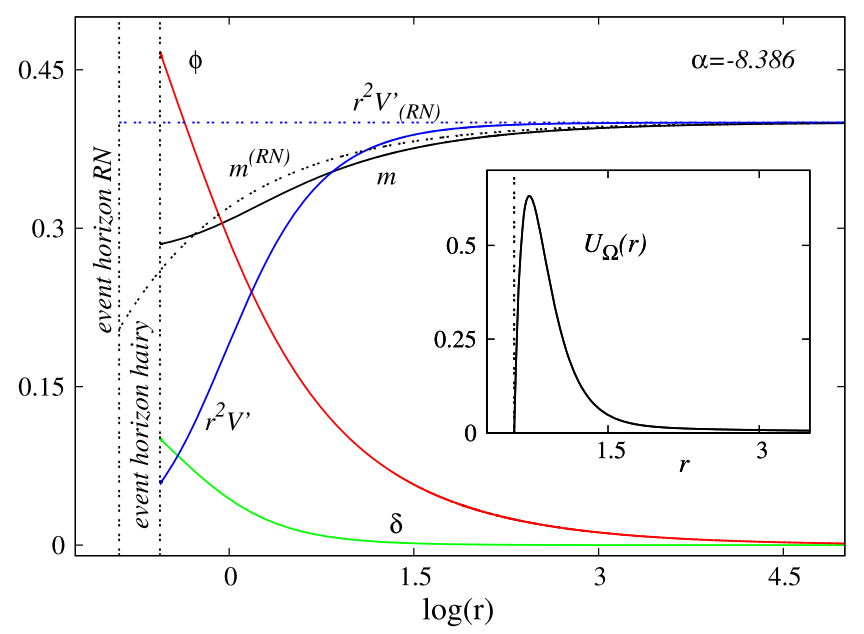

FIG. 2. Profiles for a typical spherical scalarizsed BH (solid lines) and the RN BH (dotted lines) with the same $M=0.4002$ and $Q=0.4$. The horizon areal radius is smaller for the $\mathrm{RN} \mathrm{BH}$. The inset shows the corresponding potential $U_{\Omega}$.

and the horizon area vanishes, whereas the mass and the scalar "charge" (defined as $Q_{s} \equiv-\lim _{r \rightarrow \infty} r^{2} d \phi / d r$ ) remain finite. Along the $\alpha=$ const branch, $q$ increases beyond unity. Thus, scalarized BHs can be overcharged.

The domain of existence of scalarized BHs is bounded by (i) the existence line, and (ii) the set of all critical solutions. In a part of the domain of existence there is nonuniqueness: RN and scalarized solutions coexist with the same global charges (Figs. 1 and 2). In this region, the scalarized solutions are always entropically favored, cf. Fig. 4. These spherical scalarized BHs are candidate end points of the spherical evolution (if adiabatic) of the linearly unstable RN BHs in the EMS model. Further evidence is provided by computing spherical perturbations, with frequency $\Omega$, of the scalarized solutions. The problem reduces to a single 1D Schrödinger-like equation with the potential $U_{\Omega}=\left(N / e^{2 \delta} r^{2}\right)\left[\left(e^{\alpha \phi^{2}} Q^{2}\left[2\left(\alpha \phi+r \phi^{\prime}\right)^{2}+\alpha-1\right] / r^{2}\right)+1-\right.$ $\left.N-2 r^{2} \phi^{2}\right]$. This potential vanishes both at the BH event horizon and at infinity, being regular everywhere in between. It follows that the Schrödinger equation will have no bound states if the potential is everywhere positive. For all $n=0$ solutions analyzed, this positivity is indeed satisfied. The absence of such bound states guarantees the stability of the scalarized BHs against this class of perturbations.

A final piece of dynamical evidence that the scalarization of the RN BH leads to the solutions in Fig. 1 comes from performing fully nonlinear numerical evolutions-see Ref. [38] and the Supplemental Material [33] (Sec. II) for details, which includes Refs. [22,38-40]. We start with a RN BH and a small Gaussian perturbation for $\phi$ and monitor the scalar field energy, $E_{\phi}=\int_{r_{H}}^{\infty} n^{\alpha} n^{\beta} T_{\alpha \beta}^{\mathrm{SF}} d V$, where $T^{\mathrm{SF}}$ is the $\phi$ stress-energy tensor and $n$ is the 4 -velocity of the Eulerian observer in the $3+1$ spacetime decomposition [41]. Figure 3 shows the time evolution of 


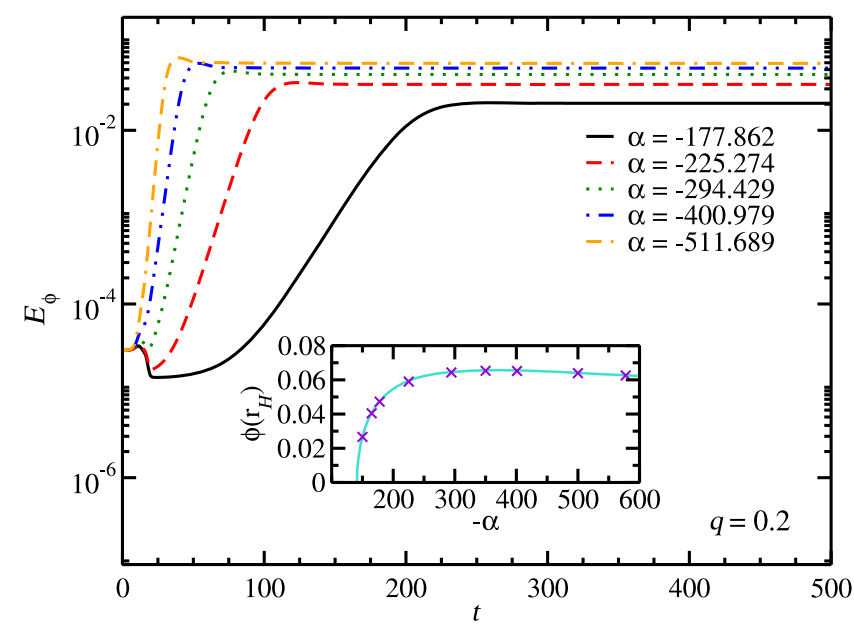

FIG. 3. Evolution of $E_{\phi}$ outside an unstable RN BH with $q=0.2$, for different couplings. Inset: Scalar field value at the horizon radius; end state of the evolutions (crosses) and static solutions (line) with $q=0.2$.

$E_{\phi}$ for an initial RN BH with $q=0.2$ and different couplings. One observes an initial growth of $E_{\phi}$ followed by a saturation and equilibrium. For the largest $|\alpha|$ saturation is faster, and it is preceded by an overshooting of the equilibrium value. The inset shows the value of the scalar field at the horizon (areal) radius, both after equilibrium is reached in each simulation (crosses) and from the scalarized BHs with the same $q=0.2$ computed as static solutions (line). The values agree within $1 \%$. Thus, the end points of the evolutions are the perturbatively stable scalarized BHs with the same $q$ as the initial unstable RN BH. A larger set of numerical evolutions, exploring the $(q, \alpha)$ space, confirms that the scalarization is generic and leads to the BHs in Fig. 1, but for $q \gtrsim 0.4$ of the initial $\mathrm{RN} \mathrm{BH}$, the evolution does not preserve $q$ (even accounting for numerical error). A discussion of these evolutions will appear elsewhere.

Non-spherical sector: Multipole freedom.-Generic $(n, \ell, m)$-scalar clouds also have nonlinear realizations, yielding branches of nonspherical scalarized BHs bifurcating from the RN trunk. We have constructed such solutions using the Einstein-De Turck approach $[42,43]$ see Ref. [44] for a review, and see the Supplemental Material [33] (Sec. III) for details, which includes Ref. [45].

All configurations constructed are regular on and outside a topologically (but not geometrically) spherical horizon and asymptotically flat. Solutions bifurcating from a scalar cloud with $m=0(m \neq 0)$ are (are not) axially symmetric. The latter, in fact, have no spatial isometries. They provide the first explicit example of static, asymptotically flat BHs without any continuous (spatial) symmetries (see Refs. [46-48] for related work).

In Fig. 4, we exhibit results concerning solutions with $(n, \ell, m)=(0,1,0) ;(0,1,1) ;(0,2,0) ;(0,2,1) ;(0,2,2) ; \quad$ and $(n, 0,0)$, for an illustrative value of the coupling $\alpha$. The latter are for $n=1,2,3$, corresponding to excited spherical
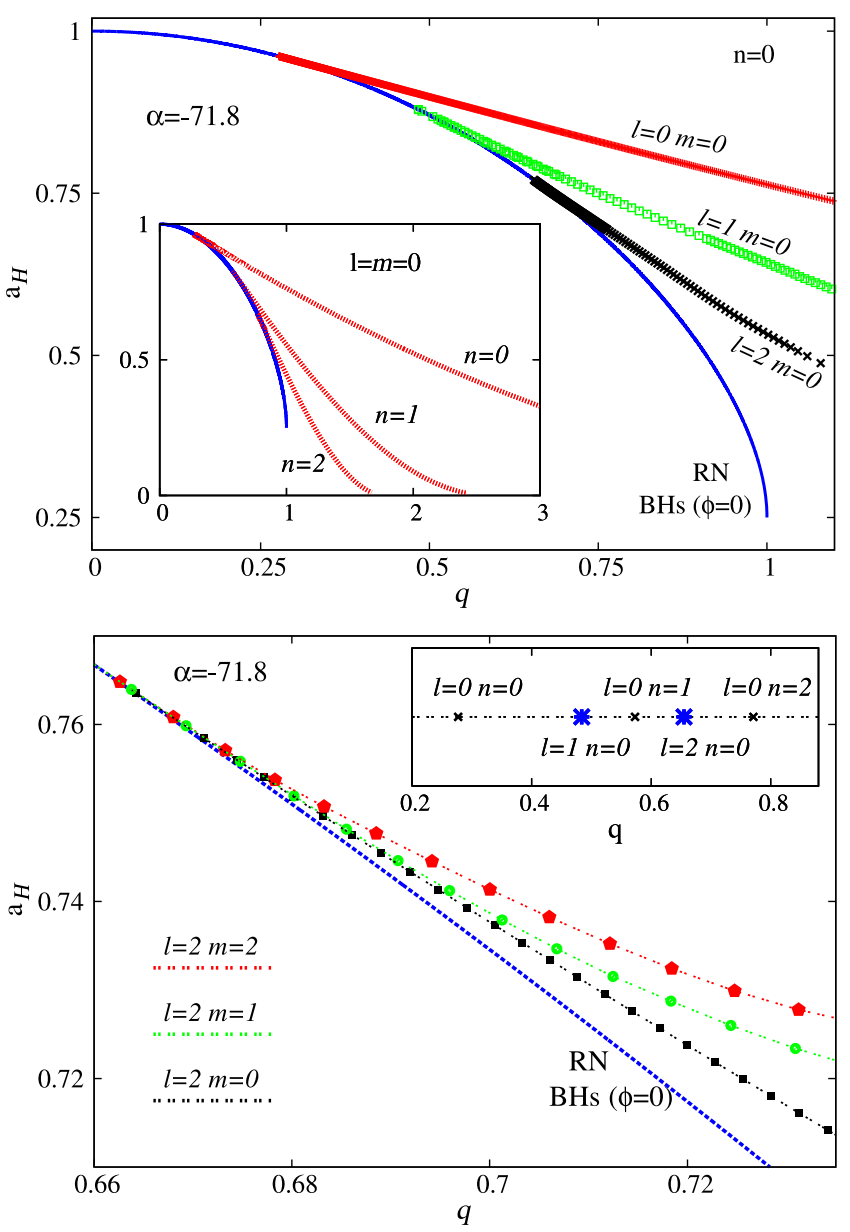

FIG. 4. Scalarized BHs with $\alpha=-71.8$, in a $q \equiv Q / M$ vs $a_{H} \equiv\left(A_{H} / 16 \pi M^{2}\right)$ diagram, where $A_{H}$ is the horizon area. BHs with $m \neq 0$ have only discrete spatial symmetries.

scalarized BHs. The top panel shows that as either $\ell$ or $n$ increases, the bifurcation point moves towards extremality of the scalar-free RN solution. This bifurcation point does not depend on $m$ (Fig. 4, bottom panel) as anticipated from the linear theory analysis. Also, the relative location of the bifurcation point for the first few values of $\ell, n$ can be seen in the inset of the bottom panel. We remark that when continuing scalar clouds with $\ell, m \neq 0$ into the nonlinear regime, the corresponding scalar field does not remain a pure $\ell, m$ mode; nonlinearities excite all $\ell$ modes with the same $m$ and parity.

All scalarized solutions are entropically preferred over a comparable RN solution (with the same total charge and mass). Within the scalarized solutions, the preferred one is the fundamental state with $\ell=0=m$, i.e., spherically symmetric. Also, for all studied cases, for constant $m$, the entropy is maximized by the branch of solutions emerging from the $\ell=m$ zero mode. These entropic arguments are, however, inconclusive for dynamical considerations.

General dynamics: The charged drop analogy.Consider a generic (i.e., nonspherical) perturbation of 
the RN BH with sufficiently large $q$. The existence of the nonspherical branches of scalarized BHs allows nonspherical perturbations to grow rather than dampen (as they would in electrovacuum). A suggestive parallelism with charged drops in fluid dynamics can be drawn.

A self-gravitating, uncharged, isolated liquid mass is spherical and stable against small perturbations [49]. But if the liquid is electrically charged, conducting and surrounded by an insulator (e.g., a gas), the competition between the cohesive tension and the electric repulsion makes the spherical drop (which remains a solution) unstable for charge beyond the Rayleigh limit [50]. New branches of nonspherical solutions of the fluid-electrostatic equations, associated with each spherical harmonic, emerge, bifurcating from the trunk family of spherical fluid balls. Such structure is analogous to that shown in Fig. 4-see, e.g., Fig. 2 in Ref. [51]. Both experiments [52] and simulations [53] show that the unstable charged drop evolves towards a nonspherical shape.

BHs in the EMS model (unlike charged drops) have a $\ell=0$ mode instability which may always dominate and lead to spherical scalarized BHs. If, however, there are initial conditions such that $\ell \neq 0$ modes dominate, a dynamical symmetry breaking may occur. Fully nonlinear numerical evolutions in the EMS model with current technology can probe this possibility.

Finally, we remark that dynamical binaries of scalarized $\mathrm{BHs}$ in this class of models will provide alternative gravitational wave templates to those of electrovacuum $\mathrm{BHs}$, in the same spirit as Ref. [22], but with the additional possibility that dynamics may trigger an initially absent scalarization.

C. H. would like to thank E. Berti for discussions. This work has been supported by the FCT (Portugal) IF programme, by Centro de Investigação e Desenvolvimento em Matemática e Aplicações (FCT) Strategic Project No. UID/MAT/04106/2013, by the Spanish MINECO (Grant No. AYA2015-66899-C2-1-P), by the Generalitat Valenciana (No. PROMETEOII-2014-069 and No. ACIF/ 2015/216), and by the European Union's Horizon 2020 research and innovation (RISE) Programmes No. H2020MSCA-RISE-2015, Grants No. StronGrHEP-690904, No. H2020-MSCA-RISE-2017, and No. FunFiCO777740. The authors would like to acknowledge networking support by the COST Action No. CA16104. Computations were performed at the Blafis cluster in Aveiro University and at the Servei d'Informàtica de la Universitat de València.

[1] B. P. Abbott et al. (Virgo and LIGO Scientific Collaborations), Phys. Rev. Lett. 116, 061102 (2016).

[2] B. P. Abbott et al. (Virgo and LIGO Scientific Collaborations), Phys. Rev. Lett. 116, 241103 (2016).
[3] B. P. Abbott et al. (Virgo and LIGO Scientific Collaborations), Phys. Rev. Lett. 118, 221101 (2017).

[4] B. P. Abbott et al. (Virgo and LIGO Scientific Collaborations), Phys. Rev. Lett. 119, 141101 (2017).

[5] B. Abbott et al. (Virgo and LIGO Scientific Collaborations), Phys. Rev. Lett. 119, 161101 (2017).

[6] B. P. Abbott et al. (Virgo and LIGO Scientific Collaborations), Astrophys. J. 851, L35 (2017).

[7] R. P. Kerr, Phys. Rev. Lett. 11, 237 (1963).

[8] B. Carter, Phys. Rev. Lett. 26, 331 (1971).

[9] D. C. Robinson, Phys. Rev. Lett. 34, 905 (1975).

[10] P. T. Chrusciel, J. L. Costa, and M. Heusler, Living Rev. Relativity 15, 7 (2012).

[11] D. D. Doneva and S. S. Yazadjiev, Phys. Rev. Lett. 120, 131103 (2018).

[12] H. O. Silva, J. Sakstein, L. Gualtieri, T. P. Sotiriou, and E. Berti, Phys. Rev. Lett. 120, 131104 (2018).

[13] G. Antoniou, A. Bakopoulos, and P. Kanti, Phys. Rev. Lett. 120, 131102 (2018).

[14] J. L. Blzquez-Salcedo, D. D. Doneva, J. Kunz, and S. S. Yazadjiev, arXiv:1805.05755.

[15] T. Damour and G. Esposito-Farese, Phys. Rev. Lett. 70, 2220 (1993).

[16] V. Cardoso, I. P. Carucci, P. Pani, and T. P. Sotiriou, Phys. Rev. Lett. 111, 111101 (2013).

[17] See, e.g., Refs. [18-21] for earlier discussions of charged BHs scalarization in different models.

[18] S. S. Gubser, Classical Quantum Gravity 22, 5121 (2005).

[19] I. Z. Stefanov, S. S. Yazadjiev, and M. D. Todorov, Mod. Phys. Lett. A 23, 2915 (2008).

[20] M. Cadoni, G. D’Appollonio, and P. Pani, J. High Energy Phys. 03 (2010) 100.

[21] D. D. Doneva, S. S. Yazadjiev, K. D. Kokkotas, and I. Z. Stefanov, Phys. Rev. D 82, 064030 (2010).

[22] E. W. Hirschmann, L. Lehner, S. L. Liebling, and C. Palenzuela, Phys. Rev. D 97, 064032 (2018).

[23] F.-L. Julié, J. Cosmol. Astropart. Phys. 01 (2018) 026.

[24] E. Barausse, C. Palenzuela, M. Ponce, and L. Lehner, Phys. Rev. D 87, 081506 (2013).

[25] M. Shibata, K. Taniguchi, H. Okawa, and A. Buonanno, Phys. Rev. D 89, 084005 (2014).

[26] C. Palenzuela, E. Barausse, M. Ponce, and L. Lehner, Phys. Rev. D 89, 044024 (2014).

[27] W. Israel, Commun. Math. Phys. 8, 245 (1968).

[28] J. D. Bekenstein, Phys. Rev. D 51, R6608 (1995).

[29] C. A. R. Herdeiro and E. Radu, Int. J. Mod. Phys. D 24, 1542014 (2015).

[30] V. Cardoso, I. P. Carucci, P. Pani, and T. P. Sotiriou, Phys. Rev. D 88, 044056 (2013).

[31] D. Garfinkle, G. T. Horowitz, and A. Strominger, Phys. Rev. D 43, 3140 (1991); 45, 3888(E) (1992).

[32] J. D. Bekenstein, Phys. Rev. Lett. 28, 452 (1972).

[33] See Supplemental Material at http://link.aps.org/ supplemental/10.1103/PhysRevLett.121.101102 for details on the numerical approach for both the stationary and time evolution problems.

[34] C. W. Misner and D. H. Sharp, Phys. Rev. 136, B571 (1964).

[35] G. H. Derrick, J. Math. Phys. 5, 1252 (1964). 
[36] M. Heusler and N. Straumann, Classical Quantum Gravity 9, 2177 (1992).

[37] M. Heusler, Helv. Phys. Acta 69, 501 (1996).

[38] N. Sanchis-Gual, J. C. Degollado, P. J. Montero, J. A. Font, and C. Herdeiro, Phys. Rev. Lett. 116, 141101 (2016).

[39] N. Sanchis-Gual, J. C. Degollado, C. Herdeiro, J. A. Font, and P. J. Montero, Phys. Rev. D 94, 044061 (2016).

[40] J. M. Torres and M. Alcubierre, Gen. Relativ. Gravit. 46, 1773 (2014).

[41] M. Alcubierre, Introduction to 3+1 Numerical Relativity (Oxford University Press, New York, 2008).

[42] M. Headrick, S. Kitchen, and T. Wiseman, Classical Quantum Gravity 27, 035002 (2010).

[43] A. Adam, S. Kitchen, and T. Wiseman, Classical Quantum Gravity 29, 165002 (2012).

[44] O. J. C. Dias, J. E. Santos, and B. Way, Classical Quantum Gravity 33, 133001 (2016).
[45] W. Schonauer and R. Weiss, J. Comput. Appl. Math. 27, 279 (1989).

[46] S. A. Ridgway and E. J. Weinberg, Phys. Rev. D 52, 3440 (1995).

[47] T. Ioannidou, B. Kleihaus, and J. Kunz, Phys. Lett. B 635 , 161 (2006).

[48] C. A. Herdeiro and E. Radu, Phys. Rev. Lett. 117, 221102 (2016).

[49] L. Rayleigh, Proc. London Math. Soc. 10, 4 (1879).

[50] L. Rayleigh, The London, Edinburgh, and Dublin Philosophical Magazine and Journal of Science 14, 184 (1882).

[51] O. A. Basaran and L. E. Scriven, Phys. Fluids A 1, 795 (1989).

[52] D. Duft, T. Achtzehn, R. Müller, B. A. Huber, and T. Leisner, Nature (London) 421, 128 (2003).

[53] J. C. Burton and P. Taborek, Phys. Rev. Lett. 106, 144501 (2011). 